\title{
Application of PLS-Regression as Downscaling Tool for Pichola Lake Basin in India
}

\author{
Manish Kumar Goyal ${ }^{*}$, Chandra Shekhar Prasad Ojha \\ Department of Civil Engineering, Indian Institute of Technology, Roorkee, India \\ E-mail:vipmkgoyal@rediffmail.com \\ Received July 12, 2010; revised July 14, 2010; accepted July 23, 2010
}

\begin{abstract}
In this paper, downscaling models are developed using Partial Least Squares (PLS) Regression for obtaining projections of mean monthly precipitation to lake-basin scale in an arid region in India. The effectiveness of this approach is demonstrated through application to downscale the predictand for the Pichola lake region in Rajasthan state in India, which is considered to be a climatically sensitive region. The predictor variables are extracted from (1) the National Centers for Environmental Prediction (NCEP) reanalysis dataset for the period 1948-2000, and (2) the simulations from the third-generation Canadian Coupled Global Climate Model (CGCM3) for emission scenarios A1B, A2, B1 and COMMIT for the period 2001-2100. The selection of important predictor variables becomes a crucial issue for developing downscaling models since reanalysis data are based on wide range of meteorological measurements and observations. In this paper, we use PLS regression for quality prediction and its use for the variable selection based on the variable importance. The results of downscaling models using PLS regression show that precipitation is projected to increase in future for $\mathrm{A} 2$ and $\mathrm{A} 1 \mathrm{~B}$ scenarios, whereas it is least for B1 and COMMIT scenarios using predictors.
\end{abstract}

Keywords: PLS Regression, Precipitation, VIP Score

\section{Introduction}

A general circulation model is a numerical mathematical model that gives the analysis of atmosphere in all three spatial dimensions based on conservation laws of momentum, energy and water vapor. These models are the most reliable tool for estimating the changes in the climate. These are also known as global climate models, generally abbreviated as GCMs. These are mathematical representations of atmospheric and oceanic properties and processes that help describe the earth's climate system $[1,2]$. However, in most climate change impact studies, such as hydrological impacts of climate change, impact models are usually required to simulate sub-grid scale phenomenon and therefore require input data at similar sub-grid scale. The methods used to convert GCM outputs into local meteorological variables required for reliable hydrological modeling are usually referred to as "downscaling" techniques: [3,4]. Hydrologic variables, such as precipitation, etc., are significant parameters for climate change impact studies. A proper assessment of probable future precipitation and their variability are to be made for various hydro-climatology scenarios.
A number of papers have previously reviewed downscaling concepts and recently, downscaling has found wide application in hydro-climatology for scenario construction and simulation/prediction of 1) low-frequency rainfall events [5] 2)streamflow [6] 3) precipitation [7] 4) streamflow [8].

In this paper, we present a downscaling methodology based on partial least square (PLS) regression technique to study climate change impact over Pichola lake basin in an arid region. The objective of this study is to obtain 1) predictor selection based on Variable Importance in the Projection (VIP) score 2) downscale mean monthly precipitation using PLS-regression approach from simulations of CGCM3 for latest IPCC scenarios. The scenarios which are studied in this paper are relevant to Intergovernmental Panel on Climate Change's (IPCC's) fourth assessment report (AR4) which was released in 2007.

\section{Study Region}

The area of the this study is the Pichola lake catchment in Rajasthan state in India that is situated from $72.5^{\circ} \mathrm{E}$ to $77.5^{\circ} \mathrm{E}$ and $22.5^{\circ} \mathrm{N}$ to $27.5^{\circ} \mathrm{N}$. The Pichola lake basin, located in Udaipur district, Rajasthan is one of the major 
sources for water supply for this arid region. During the past several decades, the streamflow regime in the catchment has changed considerably, which resulted in water scarcity, low agriculture yield and degradation of the ecosystem in the study area. Regions with arid and semi-arid climates could be sensitive even to insignificant changes in climatic characteristics. Understanding the relationships among the hydrologic regime, climate factors, and anthropogenic effects are important for the sustainable management of water resources in the entire catchment hence this study area was chosen because of aforementioned reasons. It receives an average annual precipitation of $608 \mathrm{~mm}$ based on data available from 1975-2000. It has a tropical monsoon climate where most of the precipitation is confined to a few months of the monsoon season. The location map of the study region is shown in Figure 1.

\section{Data Extraction}

The monthly mean atmospheric variables were derived from the National Center for Environmental Prediction (NCEP/NCAR) (hereafter called NCEP) reanalysis data set [9] for a period of January 1948 to December 2000. The data have a horizontal resolution of $2.5^{\circ}$ latitude $\mathrm{X}$ longitude and seventeen constant pressure levels in vertical. The atmospheric variables are extracted for nine grid points whose latitude ranges from 22.5 to $27.5^{\circ} \mathrm{N}$, and longitude ranges from 72.5 to $77.5^{\circ} \mathrm{E}$ at a spatial resolution of $2.5^{\circ}$. The meteorological data, i.e., precipitation are used at monthly time scale from records available for Pichola Lake which is located in Udaipur at $24^{\circ}$ $34^{\prime} \mathrm{N}$ latitude and $73^{\circ} 40^{\prime} \mathrm{E}$ longitude. The data is available for the period January 1975 to December 2000 [10]. The Canadian Center for Climate Modeling and Analysis
(CCCma) provides GCM data for a number of surface and atmospheric variables for the $\mathrm{CGCM} 3 \mathrm{~T} 47$ version which has a horizontal resolution of roughly $3.75^{\circ}$ latitude by $3.75^{\circ}$ longitude and a vertical resolution of 31 levels. CGCM3 is the third version of the CCCMA Coupled Global Climate Model which makes use of a significantly updated atmospheric component AGCM3 and uses the same ocean component as in CGCM2. The data comprise of present-day (20C3M) and future simulations forced by four emission scenarios, namely A1B, A2, B1 and COMMIT. Data was obtained for CGCM3 climate of the 20th Century (20CM3) experiments used in this study.

The nine grid points surrounding the study region are selected as the spatial domain of the predictors to adequately cover the various circulation domains of the predictors considered in this study. The GCM data is regridded to a common $2.5^{\circ}$ using inverse square interpolation technique. The utility of this interpolation algorithm was examined in previous downscaling studies [7,8]. The development of downscaling models for e predictand variable precipitation begins with selection of potential predictors followed by application of PLS regression on downscaling model. The developed model is then used to obtain projections of precipitation from simulations of CGCM3.

\section{Introduction to Partial Least Square Regression and Selection of Predictors}

\subsection{Partial Least Square Regression}

Partial least squares (PLS) regression is used to describe the relationship between multiple response variables and

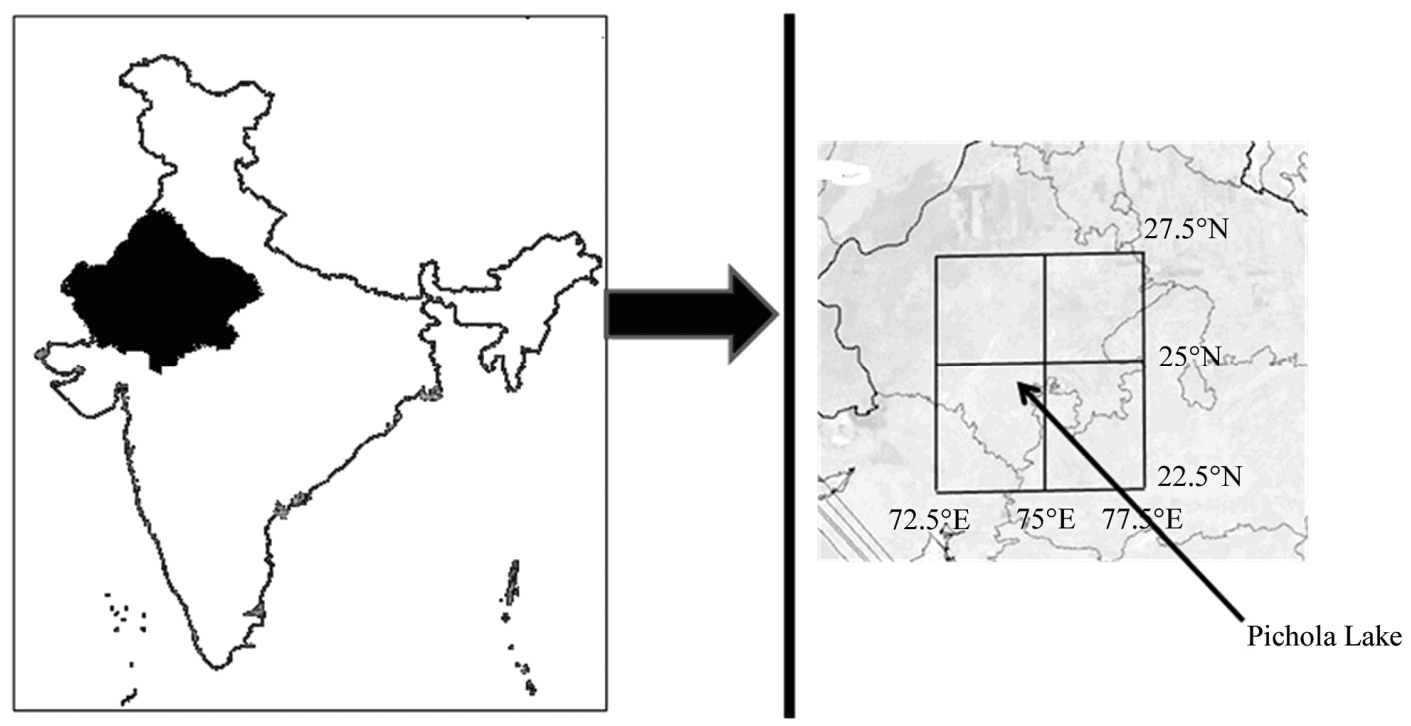

Figure 1. Location map of the study region in Rajasthan State of India with NCEP grid. 
predictors through the latent variables. PLS regression can analyze data with strongly collinear, noisy, and numerous $X$-variables, and also simultaneously model several response variables, $Y$. In general, the PLS approach is particularly useful when one or a set of dependent variables (or time series) need to be predicted by a large set of predictor variables (or time series) that are strongly cross-correlated. This is often the case in empirical downscaling of climate variables [11]. For details of PLS regression, one can refer to Manne [12] and Wold [13].

\subsection{Selections of Predictors}

The selection of appropriate predictors is one of the most important steps in a downscaling exercise for downscaling predictands. The predictors are chosen by the following criteria: 1) predictors are skillfully predicted by GCMs 2) they should represent important physical processes in the context of the enhanced greenhouse effect 3) they should not be strongly correlated to each other [14]. We have used 9 large-scale atmospheric variables, viz, air temperature (at 925,500 and $200 \mathrm{mb}$ pressure levels), geopotential height (at 200 and $500 \mathrm{mb}$ pressure levels), zonal (u) and meridional (v) wind velocities (at 925 and $200 \mathrm{mb}$ pressure levels), as the predictors for downscaling GCM output to mean monthly precipitation over a catchment.

The VIP (Variable Importance in the Projection) scores obtained by the PLS regression, has been paid an increasing attention as an importance measure of each explanatory variable or predictor [15]. The variable selection procedure under PLS is proposed with an application to downscaling technique for identifying influencing variables on understand the impact of climate change. The VIP scores which are obtained by PLS regression, can be used to select most influential variables or predictors, $X$ [15]. The VIP score can be estimated for j-th $X$-variable by

$$
V I P_{j}=\sqrt{\frac{p}{\sum_{i=1}^{k} R_{d}\left(Y, t_{i}\right)} \sum_{i=1}^{k} R_{d}\left(Y, t_{i}\right) w_{i j}^{2}}
$$

where $R_{d}$ is defined as the mean of the squares of the correlation coefficients $(R)$ between the variables and the component.

$$
R d(X, c)=\frac{1}{p} \sum_{i=1}^{k} R^{2}\left(x_{j}, c\right)
$$

Usually the predictor variable whose VIP score is greater than 0.8 and above is considered as an important variable [16]. It can be seen form Figure 2 that seven predictor variables namely air temperature at $925 \mathrm{mb}$, $500 \mathrm{mb}$ and $200 \mathrm{mb}$, zonal wind $(925 \mathrm{mb})$; meridoinal wind $(925 \mathrm{mb})$; zeo-potential height $500 \mathrm{mb}$ and $200 \mathrm{mb}$ have their VIP score greater than 0.8 . Hence, these variables are used in the prediction model to obtain projected predictands.

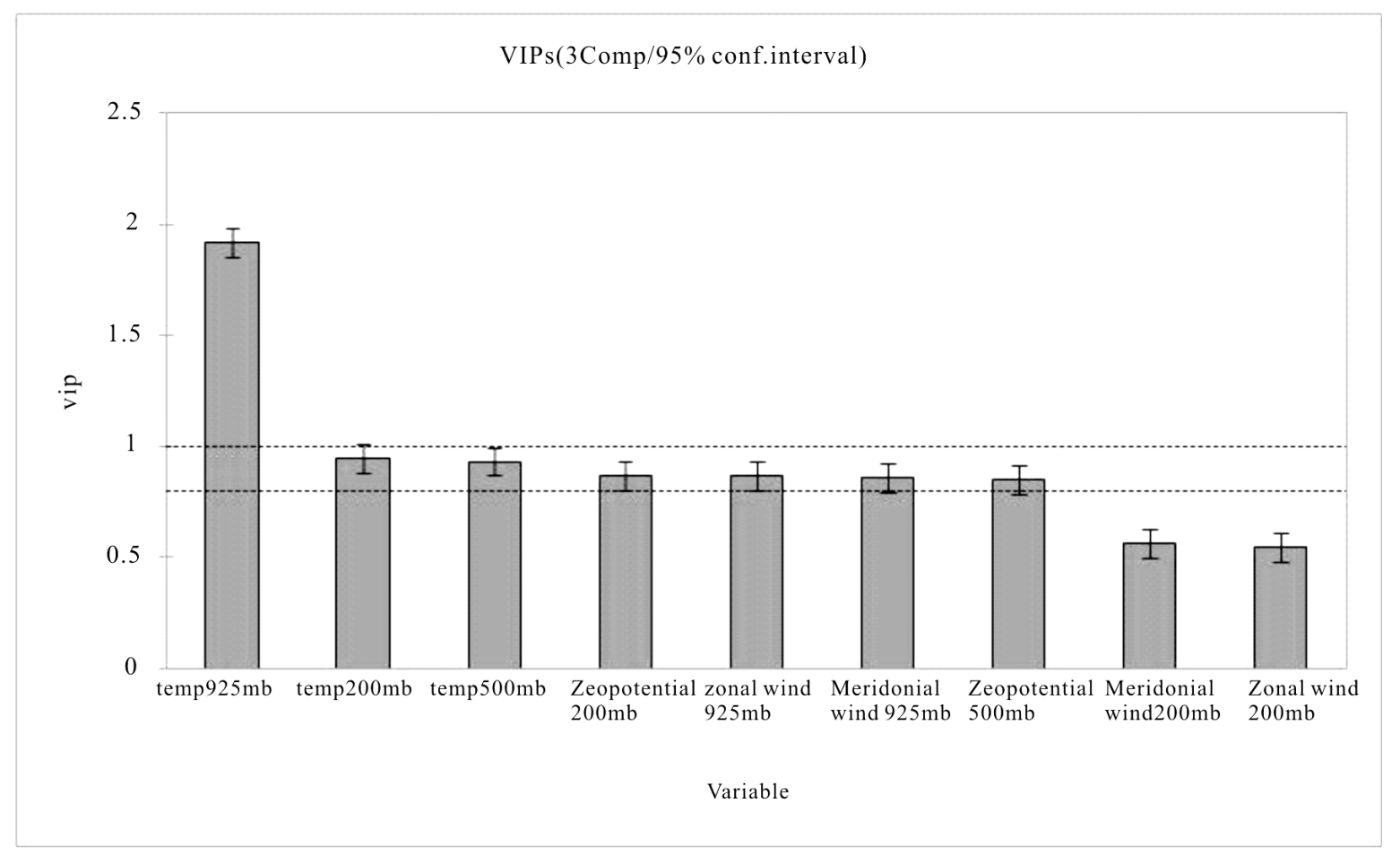

Figure 2. VIP of the predictand variable (precipitation) of the three-component PLSR model. 


\section{Downscaling of GCM Models}

There are several different methods, which can be used to derive the relationship between local and large-scale climates. PLS regression is used to downscale mean monthly precipitation in this study. The data of potential predictors is first standardized. Standardization is widely used prior to statistical downscaling to reduce bias (if any) in the mean and the variance of GCM predictors with respect to that of NCEP-reanalysis data [17]. Standardization is done for a baseline period of 1948 to 2000 because it is of sufficient duration to establish a reliable climatology, yet not too long, nor too contemporary to include a strong global change signal $[8,17]$.

To develop downscaling models, the feature vectors (i.e., predictors) which are prepared from NCEP record, are partitioned into a training set and a validation set. Feature vectors in the training set are used for calibrating the model, and those in the validation set are used for validation. The 26 -year mean monthly observed precipitation data series were broken up into a calibration period and a validation period. The models were calibrated on the calibration period 1975 to 1989 and validation involved period 1990 to 2000 . The various error criteria are used as an index to assess the performance of the model. Based on the latest IPCC scenario, models for mean monthly precipitation were evaluated based on the accuracy of the predictions for validation data set. The following criteria of PLS regression models were chosen in this study.

1) The $Q^{2}{ }_{C U M}$ index measures the global contribution of the $\mathrm{h}$ first components to the predictive quality of the model. The $Q^{2}{ }_{C U M}(h)$ index writes:

$$
Q_{C U M}^{2}=1-\sum_{j=1}^{h} \frac{P_{R E S S_{j}}}{R R S_{j-1}}
$$

where $P R E S S_{j}$ PRESS being associated with a $j$-component PLS model and $R R S_{j-1} R R S$ being associated with a $(j-1)$ component PLS model. It must be as close as possible to 1 .

2) The $R^{2} X_{C U M}$ index is the sum of the coefficients of determination between the explanatory variables and the $\mathrm{h}$ first components. It is therefore a measure of the explanatory power of the $\mathrm{h}$ first components for the explanatory variables of the model.

$$
R X_{h}^{2}=\frac{\sum_{i=1}^{h}\left\|t_{i}\right\|^{2}\left\|p_{i}\right\|^{2}}{\left(n_{L}-1\right) p} * 100
$$

3) The $R^{2} Y_{C U M}$ index is the sum of the coefficients of determination between the dependent variables and the $h$ first components. It is therefore a measure of the explanatory power of the $h$ first components for the de- pendent variables of the model.

$$
\begin{gathered}
R Y_{h, a d j}^{2}=\left[1-\left(1-R Y_{h}^{2}\right)\left(\frac{\left.n_{L}-1\right)}{n_{L}-h-1}\right)\right] * 100 \\
R Y_{h}^{2}=\frac{\sum_{i=1}^{n_{L}}\left(y_{i}-\hat{y}_{i}^{[h]}\right)}{\sum_{i=1}^{n_{L}}\left(y_{i}-\bar{y}_{i}\right)^{2}}
\end{gathered}
$$

where $y_{i}^{[h]}$ is the prediction of the observation $y_{i}$ for an $h$-component model and $y$ the average of the $n_{L}$ observations, $y_{i}$.

A comparison of proposed downscaling method with the commonly used principal component regression was performed. The leading principal components, which together explain about $98 \%$ of predictor's variability, were retained to be used in empirical model (PCRM2) development. The same error criteria were used to assess the performance of model.

\section{Results and Discussions}

Seven predictor variables namely air temperature at 925 $\mathrm{mb}, 500 \mathrm{mb}$ and $200 \mathrm{mb}$; zonal wind $(925 \mathrm{mb})$; meridoinal wind $(925 \mathrm{mb})$; zeo-potential height $500 \mathrm{mb}$ and 200 $\mathrm{mb}$ at 9 NCEP grid points with a dimensionality of 63 , are used as the standardized data of potential predictors. These feature vectors are provided as input to the PLS regression and PCR downscaling models. PLS regression is performed on this dataset. Results of the PLS regression model (viz. PLSRM1) and Principal Component based regression model (viz. PLSRM2) are tabulated in Table 2.

Model quality indexes $Q^{2}{ }_{C U M}$ index, $R^{2} X_{C U M}$ and $R^{2} Y_{\text {CUM }}$ index have been shown in Table 1. It is clear that all three indexes are highest for the three component of for predictand precipitation. $Q^{2}{ }_{C U M}$ index, $R^{2} X_{C U M}$ and $R^{2} Y_{C U M}$ index are $0.647,0.724$ and 0.907 , respectively. Hence, model quality can be considered as good.

Coefficient of correlation (CC) was in the range of $0.80-0.87$, RMSE was in the range of 43.20-44.98, N-S Index was in the range of $0.58-0.75$ and MAE was in the range of $0.44-0.58$ for PLS regression based model PLSRM1 for training and validation set. For PCRM2, Coefficient of correlation (CC) was in the range of 0.420.61 , RMSE was in the range of 78.18-120.45, N-S Index was in the range of 0.31-0.10 and MAE was in the range of $-0.16-0.19$ for PCR based model PCRM2 for training and validation set. Hence it is clear from Table 2 that PLS regression is performed better than principal component regression. A comparison of mean monthly observed precipitation with precipitation simulated using PLS regression models PLSRM1 has been shown from Figure 3 for validation period.

Once the downscaling models have been calibrated 
and validated, the next step is to use these models to downscale the control scenario simulated by the GCM. The GCM simulations are run through the calibrated and validated PLS regression model (viz. PLSRM1) to obtain future simulations of predictand. The predictand (viz. precipitation) patterns are analyzed with box plots for 20 year time slices. The middle line of the box gives the median whereas the upper and lower edges give the 75

Table 1. Various quality measures of PLS regression model (PLSRM1).

\begin{tabular}{lccc}
\hline & \multicolumn{3}{c}{ Precipitation (PLSRM1) } \\
\cline { 2 - 4 } Index & Comp1 & Comp2 & Comp3 \\
\hline$Q^{2}{ }_{\text {CUM }}$ & 0.468 & 0.633 & 0.647 \\
$R^{2} X_{\text {CUM }}$ & 0.486 & 0.659 & 0.724 \\
$R^{2} Y_{\text {CUM }}$ & 0.747 & 0.884 & 0.907 \\
\hline
\end{tabular}

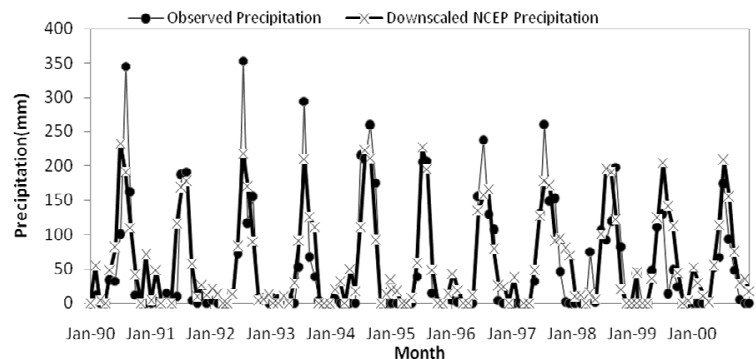

Figure 3. Typical results for comparison of the monthly observed precipitation with precipitation simulated using PLR regression downscaling model PLSRM1 for NCEP data.
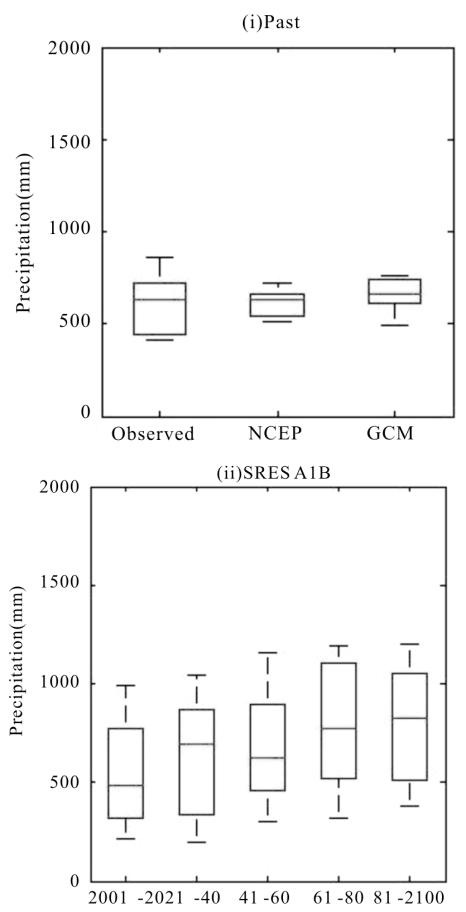
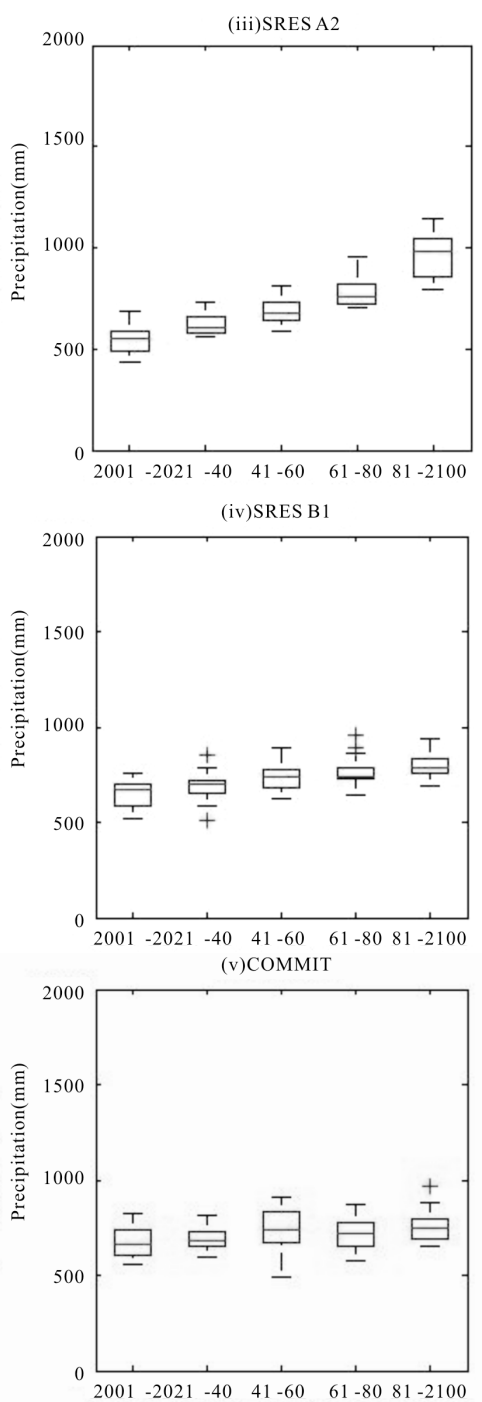

Figure 4. Box plots results from the PLS regression-based downscaling model PLSRM1 for the predictand precipiation.

percentile and 25 percentile of the data set, respectively. Typical results of downscaled predictand (precipitation) obtained from the predictors are presented in Figure 4. In part (i) of Figure 4, the precipitation downscaled using NCEP and GCM datasets are compared with the observed precipitation for the study region using box plots. The projected precipitation for 2001-2020, 2021-2040, 2041-2060, 2061-2080 and 2081-2100 for the four scenarios A1B, A2, B1 and COMMIT are shown in (ii), (iii), (iv) and (v) respectively.

From the box plots of downscaled predictand (Figure 4), it can be observed that precipitation are projected to increase in future for A1B, A2 and B1 scenarios. The average value of observed precipitation for last 26 years (1975-2000) is 608 millimeters. The average value of precipitation for 100 years (2001-2100) from SRES A1B scenario of CCCma is 677 millimeters while average 
Table 2. Various performance statistics of model PLSRM1 and PCRM2.

\begin{tabular}{|c|c|c|c|c|c|c|c|c|c|c|c|c|c|c|}
\hline \multirow[b]{2}{*}{ Model } & \multicolumn{2}{|c|}{ CR } & \multicolumn{2}{|c|}{ SSE } & \multicolumn{2}{|c|}{ MSE } & \multicolumn{2}{|c|}{ RMSE } & \multicolumn{2}{|c|}{ NMSE } & \multicolumn{2}{|c|}{ N-S Index } & \multicolumn{2}{|c|}{ MAE } \\
\hline & Training & Validation & Training & Validation & Training & Validation & Training & Validation & $\begin{array}{c}\text { Train- } \\
\text { ing }\end{array}$ & $\begin{array}{l}\text { Valida- } \\
\text { tion }\end{array}$ & Training & $\begin{array}{c}\text { Valida- } \\
\text { tion }\end{array}$ & $\begin{array}{c}\text { Train- } \\
\text { ing }\end{array}$ & $\begin{array}{c}\text { Valida- } \\
\text { tion }\end{array}$ \\
\hline PLSRM1 & 0.87 & 0.80 & 145691.44 & 111969.46 & 2023.49 & 1866.16 & 44.98 & 43.20 & 0.25 & 0.41 & 0.75 & 0.58 & 0.58 & 0.44 \\
\hline PCRM2 & 0.61 & 0.42 & 311122.00 & 451324.00 & 6112.00 & 14509.00 & 78.18 & 120.45 & 0.05 & 0.03 & 0.31 & 0.10 & -0.16 & 0.19 \\
\hline
\end{tabular}

value of precipitation for 100 years (2001-2100) from SRES A2 scenario of CCCma is 719 millimeters. The mean value of precipitation for 100 years $(2001-2100)$ from SRES B1 scenario of CCCma is 626 millimeters while mean value of precipitation for 100 years (20012100) from COMMIT scenario of CCCma is 618 millimeters. Hence, it is clear that the projected increase of precipitation is high for $\mathrm{A} 1 \mathrm{~B}$ and $\mathrm{A} 2$ scenarios whereas it is least for B1 scenario.

This is because the scenario $\mathrm{A} 1 \mathrm{~B}$ and $\mathrm{A} 2$ have the highest concentration of atmospheric carbon dioxide $\left(\mathrm{CO}_{2}\right)$ equal to $720 \mathrm{ppm}$ and $850 \mathrm{ppm}$, while the same for $\mathrm{B} 1$ and COMMIT scenarios are $550 \mathrm{ppm}$ and $\approx 370$ ppm respectively. Rise in concentration of atmospheric $\mathrm{CO}_{2}$ in the atmosphere causes the earth's average temperature to increase, which in turn causes increase in evaporation especially at lower latitudes. The evaporated water would eventually precipitate [17]. In the COMMIT scenario, where the emissions are held the same as in the year 2000, no significant trend in the pattern of projected future precipitation could be discerned. The overall results show that the projections obtained for precipitation are indeed robust.

\section{Conclusions}

This paper investigates the suitability of partial least square regression approach to downscale mean monthly precipitation from GCM output to local scale. The effectiveness of this model is demonstrated through the application of lake catchments in arid region in India. The predictands are downscaled from simulations of CGCM3 for four IPCC scenarios namely SRES A1B, A2, B1 and COMMIT.

The selection of relevant predictors used for empirical model development plays a crucial role. PLS regression has been applied for selection of important variables which have a VIP score greater than 0.8 . PLS regression seems to be a useful tool for downscaling. PLS regression seems to be a useful alternative to the commonly used PCR method for empirical downscaling. The results of downscaling models using PLS regression show that precipitation is projected to increase in future for A2 and A1B scenarios, whereas it is least for B1 and COMMIT scenarios using predictors.

\section{References}

[1] R. Weisse and R. Oestreicher, "Reconstruction of Poten- tial Evaporation for Water Balance Studies," Climate Research, Vol. 16, No. 2, 2001, pp. 123-131.

[2] C. Prudhomme, D. Jakob and C. Svensson, "Uncertainty and Climate Change Impact on the Flood Regime of Small UK Catchments," Journal of Hydrology, Vol. 277, No. 1, 2003, pp. 1-23.

[3] R. L. Wilby, C. W. Dawson and E. M. Barrow, "SDSM A Decision Support Tool for the Assessment of Climate Change Impacts," Environmental Modelling \& Software, Vol. 17, No. 2, 2002, pp. 147-159.

[4] M. K. Goyal and C. S. P. Ojha, "Robust Weighted Regression as a Downscaling Tool in Temperature Projections," International Journal of Global Warming. http:// www.inderscience.com/browse/index.php?journalID $=331$ \&action=coming

[5] R. L. Wilby, "Modelling Low-Frequency Rainfall Events Using Airflow Indices, Weather Patterns and Frontal Frequencies," Journal of Hydrology, Vol. 213, No.1-4, 1998, pp. 380-392.

[6] A. J. Cannon and P. H. Whitfield, "Downscaling Recent Streamflow Conditions in British Columbia, Canada Using Ensemble Neural Network Models," Journal of Hydrology, Vol. 259, No. 1, 2002, pp. 136-151.

[7] S. Tripathi, V. V. Srinivas and R. S. Nanjundiah, "Downscaling of Precipitation for Climate Change Scenarios: A Support Vector Machine Approach," Journal of Hydrology, Vol. 330, No. 3-4, 2006, pp. 621-640.

[8] S. Ghosh and P. P. Mujumdar, "Statistical Downscaling of GCM Simulations to Streamflow Using Relevance Vector Machine," Advances in Water Resources, Vol. 31, No. 1, 2008, pp. 132-146.

[9] E. Kalnay, et al., "The NCEP/NCAR 40-Year Reanalysis Project," Bulletin of the American Meteorological Society, Vol. 77, No. 3, 1996, pp. 437-471.

[10] S. D. Khobragade, "Studies on Evaporation from Open Water Surfaces in Tropical Climate," PhD Dissertation, Indian Institute of Technology, Roorkee, 2009.

[11] K. Bergant and L. K. Bogataj, "N-PLS Regression as Empirical Downscaling Tool in Climate Change Studies," Theoretical and Applied Climatology, Vol. 81, No. 1-2, 2005, pp. 11-23.

[12] R. Manne, "Analysis of Two Partial Least Squares Algorithms for Multivariate Calibration," Chemometrics and Intelligent Laboratory Systems, Vol. 2, No. 1, 1987, pp. 187-197.

[13] W. Svante, M. Sjostrom and L. Eriksson, "PLS-Regression: A Basic Tool of Chemometric," Chemometrics and Intelligent Laboratory Systems, Vol. 58, No. 2, 2001, pp. 109-130.

[14] B. C. Hewitson and R. G. Crane, "Climate Downscaling: 
Techniques and Application," Climate Research, Vol. 7, 1996, pp. 85-95.

[15] I. G. Chong and C. H. Jun, "Performance of Some Variable Selection Methods When Multicollinearity is Present," Chemometrics and Intelligent Laboratory Systems, Vol. 78, No. 1-2, 2005, pp. 103-112.

[16] L. Eriksson, E. Johansson, N. Kettaneh-Wold and S.
Wold, Multi- and Megavariate Data Analysis: Principles and Applications, Umetrics Academy, Umeå, 2001.

[17] A. Anandhi, V. V. Srinivas, D. N. Kumar, R. S. Nanjundiah, "Role of Predictors in Downscaling Surface Temperature to River Basin in India for IPCC SRES Scenarios Using Support Vector Machine," International Journal of Climatology, Vol. 29, No. 4, 2009, pp. 583-603. 